\title{
International Classification of Functioning, Disability and Health: development of an assessment set to evaluate functioning based on the Brief ICF Core Set for Hand Conditions - ICF Hand
}

The Journal of Hand Surgery (European Volume)

2017, Vol. 42E(7) 731-741 (c) The Author(s) 2017

Reprints and permissions: sagepub.co.uk/journalsPermissions.nav DOI: 10.1177/1753193417706248 journals.sagepub.com/home/jhs (SAGE

\author{
S. Kus ${ }^{1,2}$, C. Dereskewitz ${ }^{3}$, M. Coenen ${ }^{1,2}$, A. Rauch ${ }^{1}$, K.-D. Rudolf ${ }^{3}$ \\ and the Consortium Lighthouse Project Hand
}

\begin{abstract}
Timely identification of patients' problems after disorder or injury of the hand requires a thorough functional assessment. However, the variety of outcome measures available makes it difficult to choose the appropriate instrument. The brief International Classification of Functioning, Disability and Health (Brief ICF Core Set for Hand Conditions) provides a standard for what aspects need to be measured in hand injuries and disorders without specifying how to make the assessment. We developed the ICF-based Assessment Hand (ICF Hand A $_{A}$, an assessment set for functioning based on the Brief ICF Core Set for Hand Conditions. First, we performed a literature review and an expert survey to pool outcome measures appropriate to assess functioning in clinical practice. At an interdisciplinary consensus conference experts decided on the outcome measures to be included in the ICF Hand . The ICF Hand $_{A}$ provides a consensus on outcome measures and instruments to systematically assess function in patients with hand injuries and disorders.
\end{abstract}

\section{Keywords \\ International Classification of Functioning, Disability and Health, ICF, ICF Core Set, hand disorders, hand injuries, outcome assessment}

Date received: 12th July 2016; revised: 28th February 2017; accepted: 2nd April 2017

\section{Introduction}

Hand surgeons and therapists require standardized assessments (MacDermid, 2014) to timely identify patients' impairments, limitations and restrictions, and to guide treatment in a multidisciplinary setting for achieving the best result for the patient. Such assessments also provide standardized information along the continuum of care. We know from clinical practice and the literature that a multitude of assessments exist to evaluate clinical outcomes of patients with hand injuries and disorders (Changulani et al., 2008; Dubert, 2014; MacDermid, 2014; van de VenStevens et al., 2009; Velstra et al., 2011). In numerous studies, highly diverse assessments have been applied (Kus et al., 2011a). However, the lack of consensus regarding the assessments limits comparability of data across studies and hampers in-depth professional exchanges among experts.
The International Classification of Functioning, Disability and Health (ICF) (World Health Organization, 2001) is based on a biopsychosocial view of

\footnotetext{
'Department of Medical Informatics, Biometry and Epidemiology, Ludwig-Maximilians-Universität, München, Germany ${ }^{2}$ ICF Research Branch - a cooperation partner within the WHO Collaborating Centre for the Family of International Classifications in Germany (at DIMDI)

${ }^{3}$ Department of Hand Surgery, Plastic and Microsurgery, BG

Trauma Hospital, Hamburg, Germany

Corresponding author:

S. Kus, PhD, MPH, Department of Medical Informatics, Biometry and Epidemiology, Chair for Public Health and Health Services

Research, Ludwig-Maximilians-Universität München, 17

Marchioninistrasse, 81377 Munich, Germany.

Email: sandra.kusamed.lmu.de
} 
functioning, which is the outcome of the interaction between a health condition and contextual factors (environmental and personal factors). Based on this concept, the classification includes alphanumerical coded ICF categories of the components: Body Functions (b), Body Structures (s), Activities and Participation (d), and Environmental Factors (e). The Brief ICF Core Set for Hand Conditions (ICF CS-HC) (Rudolf et al., 2012) specifies functioning domains and environmental factors relevant in hand injuries and disorders. It provides a well-established and validated list, including 23 ICF categories, and serves as the minimal standard that should be applied to report on functioning and environmental factors of persons with hand injuries or disorders (Kus et al., 2012). The ICF CS-HC defines what to measure le.g. impairment of a body function) without providing information on how to measure it. This makes the set a helpful reference rather than a standardized assessment instrument for clinical use. A standardized assessment set based on the ICF CS-HC would facilitate the assessment and the comparability of functioning-related information. It could also be used in medical reports and improve communication among health professionals, service providers and cost bearers. This article describes the decision-making process used to develop the ICF-based Assessment Hand (ICF Hand ${ }_{A}$ ). The specific aims were to report on the results of the preliminary studies chosen to provide evidence for a consensus conference and to present the results of this conference.

\section{Methods}

We adopted a multistage, evidence-based process to

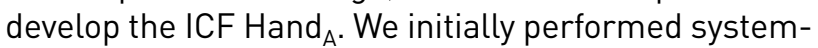
atic literature reviews and a national survey with clinical experts. The results of these studies provided information for the participants of a consensus conference who then decided on the ICF Hand ${ }_{A}$. The studies were performed within the Lighthouse Project Hand.

\section{Preliminary study: systematic literature reviews}

We carried out systematic literature reviews to identify outcome measures and instruments (hereinafter referred to as 'measures') used to assess the 23 categories of the ICF CS-HC. The literature reviews followed the PRISMA guidelines (Moher et al., 2009) (PRISMA guidelines define an evidence-based minimum set of aspects to be reported in systematic reviews and meta-analyses). We searched for standardized outcome measures, such as patient-reported outcomes (PRO), clinical assessments (e.g. hand dynamometer) and physical examinations (e.g. goniometry).

We conducted a search for every ICF category of the ICF CS-HC (e.g. 'b265 Touch function'). We searched MEDLINE, CINAHL, PsycINFO, PEDro and OTseeker for studies focusing on individuals with hand injuries or disorders that reported the use of measures (see search strategy for 'b265 Touch function' as electronic supplementary material). Randomized controlled trials, clinical controlled trials, cross-sectional and longitudinal studies, validation studies, epidemiological trials, qualitative studies and psychometric studies published between 2007 and 2012 in English or German were included. Exclusion criteria were: (1) sole inclusion of individuals with injuries and/or disorders of shoulder, elbow or upper arm; (2) ICF category of interest was not addressed in the measures used; (3) measures were not reported; (4) no abstract available. For practical reasons, whenever a search retrieved more than 1000 records after duplicate checks, we performed random sampling to obtain a $30 \%$ sample. Two researchers (SK, MC) checked the abstracts from all studies and extracted data on diagnosis, measures used for data collection and type of measures (e.g. PRO, clinical measure, etc.). Whenever there were uncertainties within the abstract regarding which specific measure the authors had used to evaluate an outcome le.g. 'sensibility'), we analysed the full text. Frequency analyses were performed on the measures that were identified in the retrieved studies.

\section{Preliminary study: expert survey}

We performed a national expert survey to identify measures used in clinical routine to assess the categories included in the ICF CS-HC. The survey was carried out as a stand-alone research study, and measures identified in the systematic review were not presented to the participants.

We recruited hand specialists from Germanspeaking countries by contacting hand clinics and centres, professional associations and authors of hand-specific publications identified by internet search (e.g. PubMed). Experts had to meet the following inclusion criteria: professional background as physician, occupational therapist, physiotherapist, hand therapist, nurse, psychologist or social worker with at least 5 years of experience in treatment, rehabilitation or care of individuals with hand injuries or disorders. Identified experts received an email with information, the invitation to participate in the survey and the request to nominate other hand specialists (snowball system) (Biernacki and Waldorf, 1981). 


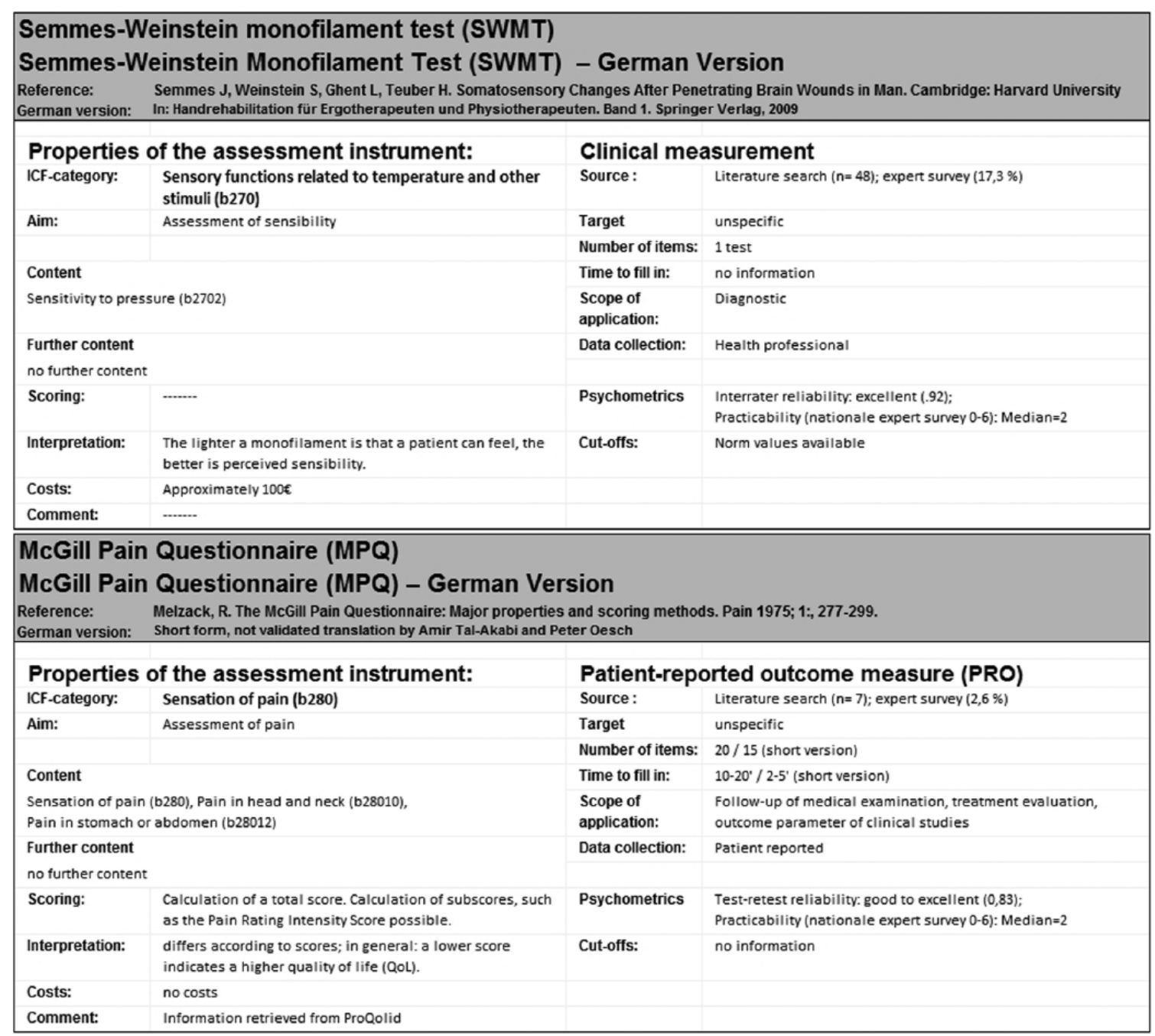

Figure 1. Consensus conference: example of materials handed out to the conference participants.

Materials: The survey questionnaire consisted of two parts. Part I contained basic information such as age, sex, professional background and years of experience as a hand specialist. Part II asked for measures the expert commonly uses in clinical routine to assess the ICF categories included in the ICF CS-HC.

Specialists agreeing to participate received an email with information about the survey, the questionnaire and instructions on how to fill it in. The time allotted to complete the questionnaire was 3 weeks. Reminders were sent immediately before and after the deadline. Parts I and II of the questionnaire were analysed descriptively. Frequency analyses were performed on the measures listed by the participants.

\section{Consensus conference}

A 2-day consensus conference (hereinafter referred to as 'conference') involving an interdisciplinary panel was held to create the ICF Hand $\mathrm{H}_{\mathrm{A}}$ Participants belonged to previously established working groups of the Lighthouse Project Hand representing clinical experts (i.e. physicians, physiotherapists, occupational therapists and psychologists working at specialized departments/clinics for hand surgery in one out of ten large hospitals in Germanyl and representatives of the German Social Accident Insurance. We applied a multistep consensus procedure to reach agreement among the experts on measures to be included in the

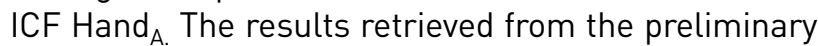
studies served as a basis for the selection. In order to be presented at the conference, measures had to be available in German language and standardization had to be described in the identified study itself or elsewhere in corresponding references. Information on psychometric properties of the measures was gained from original reference or secondary publications and was handed out to the participants in order to inform the decision-making process (see Figure 1). During 


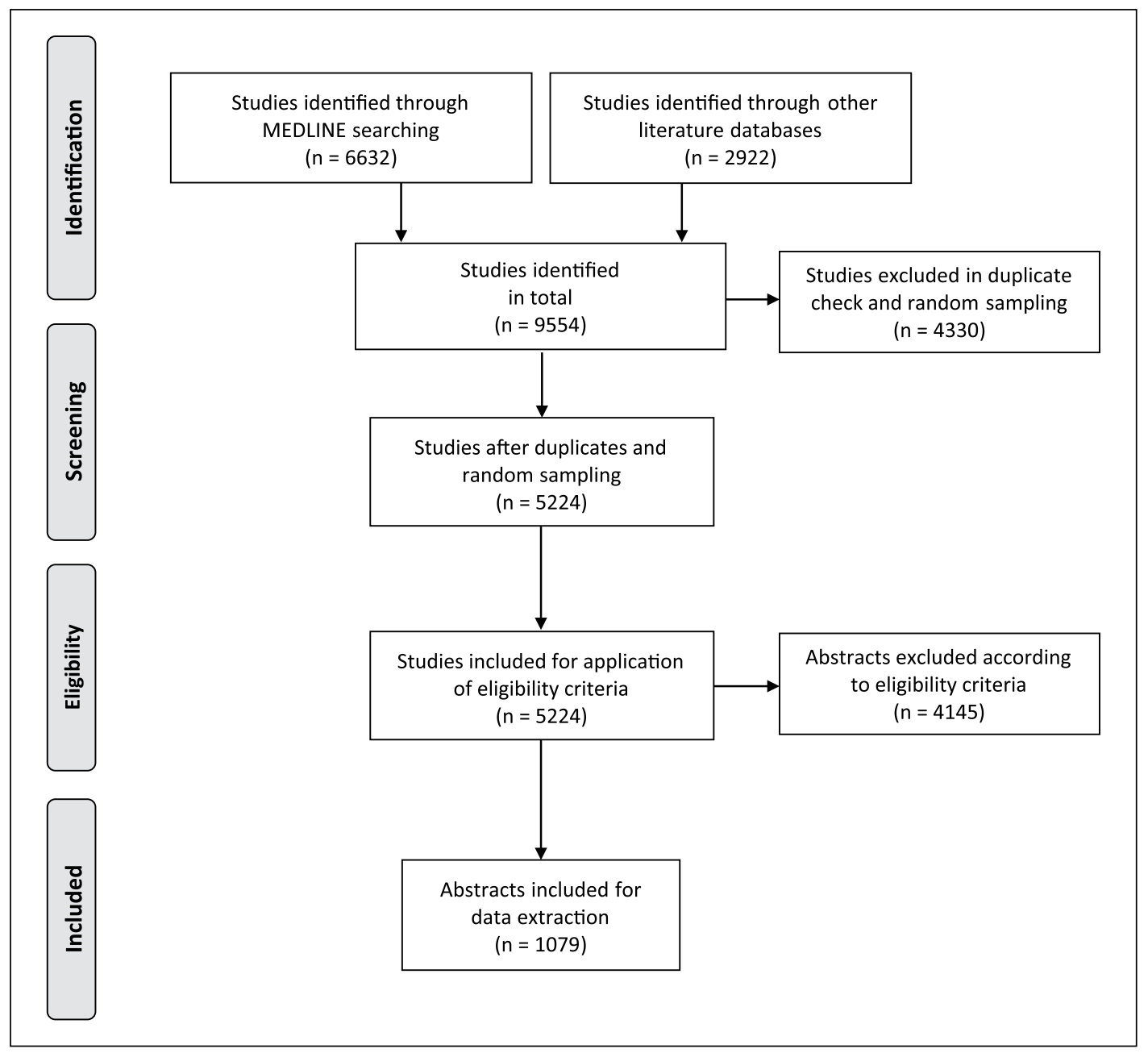

Figure 2. Systematic literature review: study selection process of the systematic review.

the consensus procedure, experts discussed and voted in profession-specific working groups, presented their decisions to the panel and performed final voting in plenary sessions to agree on the measures to be included in the ICF Hand . $_{\text {. }}$

\section{Results}

\section{Preliminary studies}

From 23 literature reviews, we identified 9554 studies. From these, 5224 abstracts were screened for eligibility and 1079 studies fulfilled the inclusion criteria. A flow chart of the selection process is shown in Figure 2. In total, 246 measures were extracted from the studies. We selected 153 measures (shown in the online appendix S4) to be presented at the conference based on the previously described criteria. Measures that existed only in English or for which standards of usage were missing were excluded.
From 923 physicians and therapists contacted, 161 $(17 \%)$ participated in the survey (Table 1). They named 121 measures, of which 99 had already been identified from the literature reviews. Consequently, we added 22 measures for the presentation at the conference (marked with an asterisk in Appendix S4).

The measures retrieved from the preliminary studies most frequently addressed the ICF categories 'd440 Fine hand use', 'b280 Sensations of pain' and 'd445 Hand and arm use' (Table 2). The disabilities of arm, shoulder and hand (DASH) Questionnaire (Hudak et al., 1996) and the Michigan Hand Outcomes Questionnaire (MHQ) (Chung et al., 1998) covered most of the aspects of the ICF CS-HC.

Consensus conference - creation of the ICF Hand

A panel consisting of 42 professionals from different disciplines attended the 2-day conference in Hamburg in November 2012. Information on participants' 
Table 1. Expert survey and consensus conference: description of participants of the national expert survey $(N=161)$ and of the 2-day consensus conference $(N=42)$.

\begin{tabular}{|c|c|c|}
\hline \multicolumn{3}{|l|}{ Expert survey $(\mathrm{N}=161)$} \\
\hline \multirow[t]{2}{*}{ Sex } & Male & $58 \%(n=93)$ \\
\hline & Female & $42 \%(n=68)$ \\
\hline Age (in years) & & $M=41.4(S D=9.77)$ \\
\hline \multirow[t]{3}{*}{ Profession } & Physicians & $42 \%(n=68)$ \\
\hline & Therapists (PT, OT, hand therapists) & $54 \%(n=87)$ \\
\hline & Others & $4 \%(n=6)$ \\
\hline $\begin{array}{l}\text { Experience in the treatment } \\
\text { of hand conditions (in years) }\end{array}$ & & $M=11.5(S D=8.35)$ \\
\hline \multicolumn{3}{|l|}{ Consensus conference ( $\mathrm{N}=42)$} \\
\hline \multirow[t]{2}{*}{ Sex } & Male & $48 \%(n=20)$ \\
\hline & Female & $52 \%(n=22)$ \\
\hline \multirow[t]{4}{*}{ Profession } & Physicians & $33 \%(n=14)$ \\
\hline & Therapists (PT, OT, hand therapists) & $36 \%(n=15)$ \\
\hline & Psychologists & $2 \%(n=1)$ \\
\hline & Insurance representatives & $19 \%(n=12)$ \\
\hline
\end{tabular}

PT: physical therapist; OT: occupational therapist.

Table 2. Frequency analysis of outcome measures retrieved from the systematic reviews and from the expert survey.

\begin{tabular}{|c|c|c|c|c|}
\hline \multicolumn{2}{|c|}{ Aspects of the Brief ICF Core Set for HC } & \multicolumn{3}{|c|}{$\begin{array}{l}\text { Number of outcome measures addressing the single } \\
\text { aspects }\end{array}$} \\
\hline & & Total & Systematic reviews & Expert survey \\
\hline b152 & Emotional functions & 25 & 20 & 11 \\
\hline b265 & Touch functions & 37 & 37 & 7 \\
\hline b270 & $\begin{array}{l}\text { Sensory functions related to temperature } \\
\text { and other stimuli }\end{array}$ & 42 & 41 & 11 \\
\hline b280 & Sensation of pain & 47 & 45 & 12 \\
\hline b710 & Mobility of joint functions & 35 & 35 & 8 \\
\hline b715 & Stability of joint functions & 7 & 7 & 4 \\
\hline b730 & Muscle power functions & 39 & 36 & 12 \\
\hline b760 & Control of voluntary movement functions & 11 & 11 & 3 \\
\hline b810 & Protective functions of the skin & 11 & 10 & 2 \\
\hline s120 & Spinal cord and related structures & 12 & 10 & 6 \\
\hline s720 & Structure of shoulder region & 1 & 1 & 1 \\
\hline s730 & Structure of upper extremity & 17 & 17 & 2 \\
\hline $\mathrm{d} 230$ & Carrying out daily routine & 7 & 5 & 5 \\
\hline d430 & Lifting and carrying objects & 25 & 22 & 8 \\
\hline d440 & Fine hand use & 50 & 48 & 12 \\
\hline d445 & Hand and arm use & 43 & 40 & 12 \\
\hline d5 & Self-care & 40 & 38 & 8 \\
\hline d6 & Domestic life & 25 & 23 & 7 \\
\hline d7 & $\begin{array}{l}\text { Interpersonal interactions and } \\
\text { relationships }\end{array}$ & 18 & 18 & 3 \\
\hline d840-d859 & Work and employment & 36 & 33 & 9 \\
\hline e1 & Products and technology & 10 & 9 & 1 \\
\hline e3 & Support and relationships & 12 & 11 & 1 \\
\hline e5 & Services, systems and policies & 5 & 4 & 1 \\
\hline
\end{tabular}

professional backgrounds is presented in Table 1. The participants decided on the ICF Hand $_{A}$ and agreed on a two-stage procedure involving a screening and a supplementary test of functioning, if indicated. They also decided to take into account the patient perspective by adding the DASH. In the screening, a physician 
guides the entire assessment of functioning by evaluating the patient's problems regarding aspects of the ICF CS-HC using the measures of the ICF Hand (Table 3; column: Screening).

For Body structures, established diagnostic imaging techniques, such as radiography, are used, if necessary, to document localization and nature of the impairments (i.e. nerves, muscles and tendons of the forearm and hand). For Body functions, PROs and various clinical measures are used to assess impairments. For example, emotional functions are rated by using standardized screening questions le.g. Generalised Anxiety Disorder-2 (Kroenke et al., 2007). Sensory function is rated by using two-point discrimination (Lundborg and Rosen, 2004). For Activities and participation, standardized questions, performance tasks and single items from the DASH are used to screen for limitation and restrictions. For example, problems in self-care are assessed by means of three DASH items, namely: 'Wash or blow dry your hair', 'Wash your back' and 'Put on a pullover sweater'. For Environmental factors, patients' further needs, such as drugs or assistive devices, were evaluated. In the electronic supplementary material, a detailed overview of

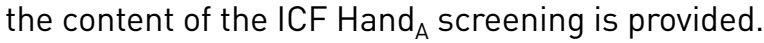

If a supplementary examination is indicated according to the results of the screening, the ICF Hand $_{A}$ provides 11 outcome measures for optional supplementary testing by capturing nine aspects of the ICF CS-HC (Table 3; column: Supplementary testingl. The supplementary test is initiated by the physician who guided the screening. Depending on the organizational structure of the individual clinic, health professionals are involved in the supplementary testing.

\section{Discussion}

We report on the multistage, evidence-based, decision-making process used to develop the ICF Hand ${ }_{A}$. This assessment is based on the ICF CS-HC, and consists of internationally established measures enabling a standardized assessment of functioning. In the systematic literature reviews we found a great number and heterogeneity of measures (Changulani et al., 2008; Goldhahn et al., 2008; Marks et al., 2013; Velstra et al., 2011) making a comparison of information and results difficult. Applying the ICF as a framework to select, classify or develop outcome measures has already been suggested (Birch et al., 2011; Bryant and Fernandes, 2011; Metcalf et al., 2007; Rosales, 2015). The aspects included in the ICF Hand $_{A}$ refer to the ICF and therefore reflect the comprehensive view of health and functioning. The ICF Hand $_{A}$ provides for the first time a set of instruments to assess these domains (Marks et al., 2013). However, in particular cases, other aspects of functioning not included in the ICF CS-HC could be relevant, especially for activities and participation (e.g. caring for others, sports).

The experts at the conference agreed on a twostage procedure - screening and the supplementary testing - to improve the clinical feasibility of the ICF Hand $_{A}$ in light of time and staff limitations in clinical practice. Depending on the current situation of a patient (e.g. time point in continuum of care), not all

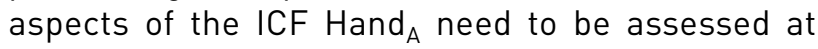
every visit. Furthermore, assessment of a functioning aspect that is obviously not impaired is dispensable with documentation of its status as no impairment' is sufficient. By applying the screening, clinicians could provide a comprehensive overview of all domains of function and identify aspects that require further testing.

All measures le.g. DASH, two-point discrimination) selected are internationally established and validated instruments. Some performance tasks le.g. Push/pull an object) or single questions (e.g. 'Do you have problems in planning, managing and completing your daily requirements?'), however, require validation in the future. Even though developed as a

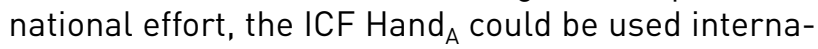
tionally after translation validation.

Implementing it in clinical practice would yield valuable and standardized information on patients' functioning irrespective of the type of injury or treatment. Standardized data on functioning could be generated for clinical research and along the continuum of care. A prospective data pool would facilitate comparability of outcomes after surgical interventions and rehabilitation. This would allow for complex data analyses, such as predictive modelling and predicting outcomes, such as return to work. This is an indispensable requirement to provide evidence on interventions or treatment efficacy. Nevertheless, to fully benefit from the advantages of using the ICF Hand $_{A}$, a user-friendly electronic data documentation tool is required, as handling data in a paper-pencil record form will be far too time-consuming.

Some methodological aspects are relevant. We did not involve patients in the decision on which measures should be included in the ICF Hand . $_{\text {. }}$ Nevertheless, the patient perspective was considered in the development process of the ICF CS-HC, on

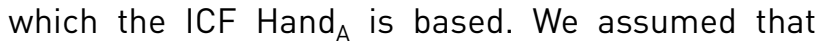
which measures are the most appropriate to be used in clinical practice required professional expertise. Representatives of the accident insurance companies, however, were invited to attend the conference to consider important aspects, such as return to work 


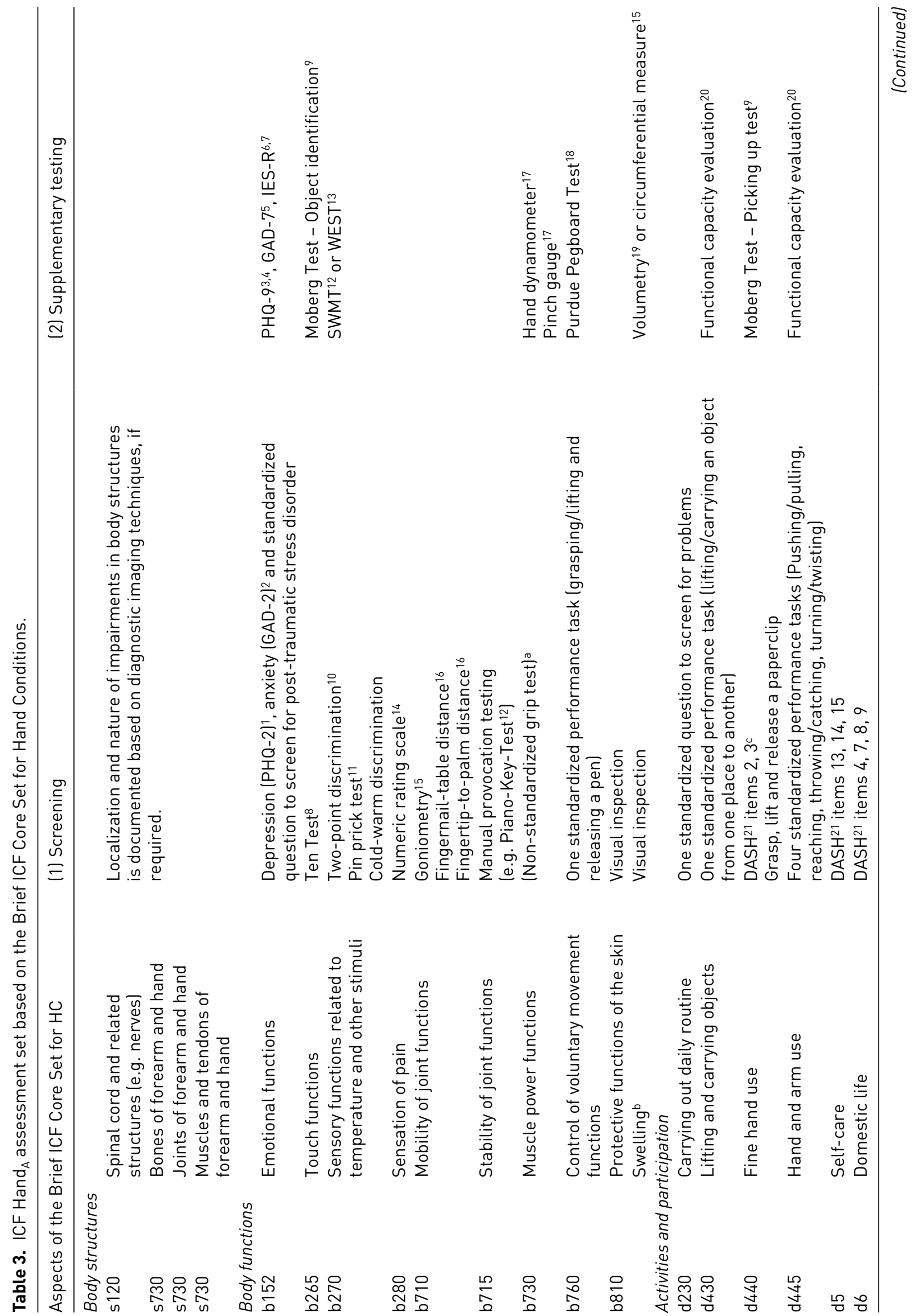




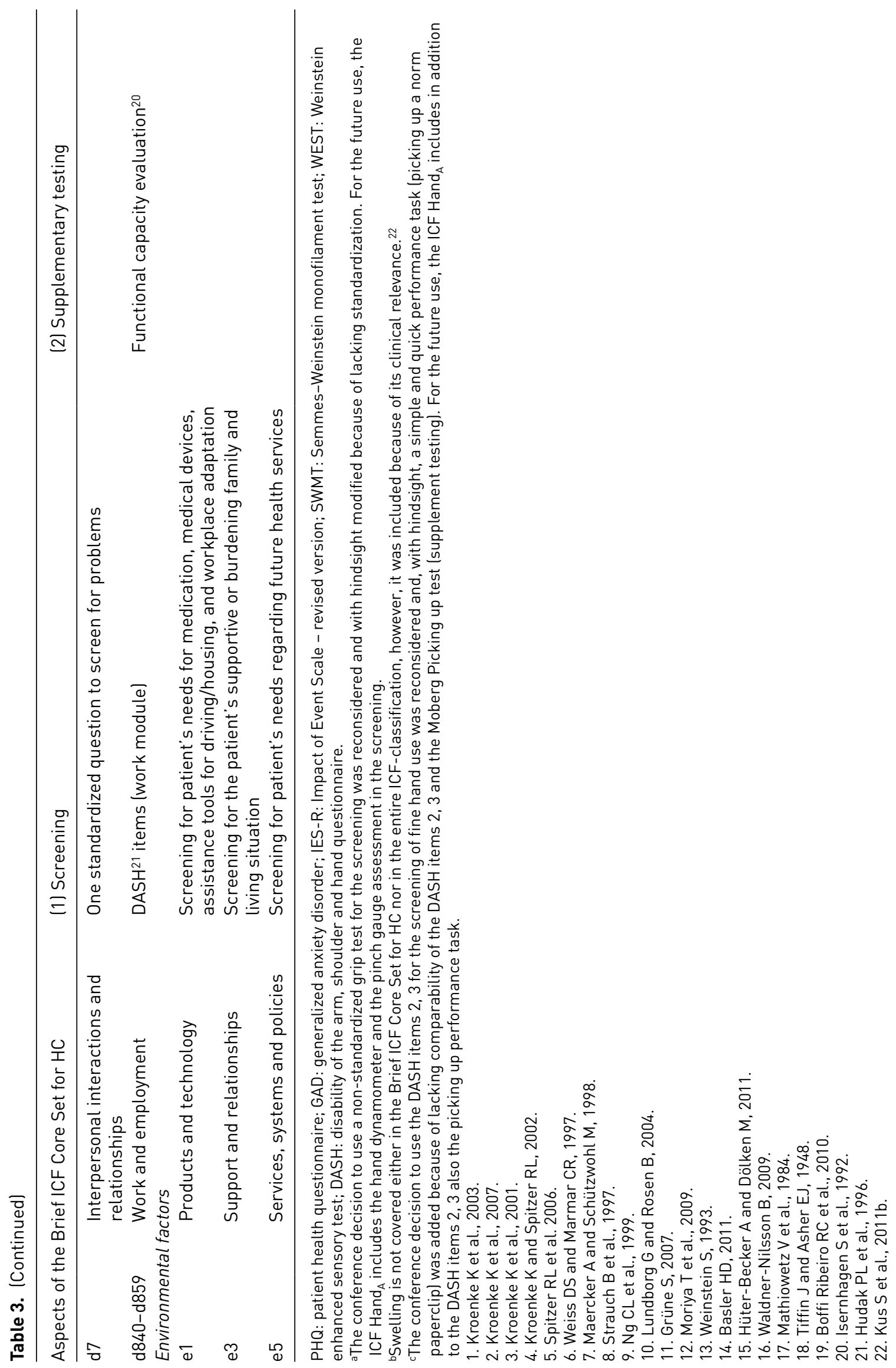


(e.g. workplace adaptations) and environmental factors (e.g. assistance tool for housingl to ensure appropriate guidance in the rehabilitation process.

In the systematic literature reviews we found that study outcomes mostly covered more than a single domain (e.g. grip strength, pain, muscle power). Thus, there was considerable overlap of studies retrieved. Likewise, the same measure could be identified more than once in various searches, as many of them, particularly PROs, include several aspects of functioning. It also became apparent that hand-specific measures addressing activities and participation primarily focus on fine hand use and exclude self-care and daily activities of domestic life. Due to this weakness and to further reflect the patient perspective, the conference participants decided to include items from the DASH to assess self-care, domestic life, work and employment, and fine hand use.

The expert survey largely confirmed the results of the literature review. Nevertheless, since 22 measures could additionally be identified, we consider the survey as indispensable.

Although applying two preliminary studies, we were only able to identify a few measures addressing environmental factors. A previous study of 260 patients emphasized the importance of environmental factors in a patient's life after having experienced an injury or disorder of the hand (Kus et al., 2012). As appropriate measures on environmental factors were lacking, the conference participants decided to add

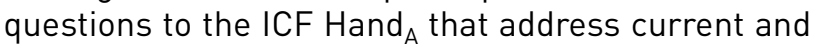
future needs for medication, medical devices, assistance tools (e.g. for driving), workplace adaptation and needs for future healthcare services in addition to questions about the patient's supportive or burdening family and living situation.

In contrast to the ICF CS-HC, the assessment of 'swelling of the hand' is part of the ICF Hand . $_{\text {. }}$ Swelling in arms and hands is generally not covered in the entire ICF classification (Kus et al., 2011a; van de Ven-Stevens et al., 2015). However, the conference included this aspect because of its importance in clinical practice. The physician rates 'swelling' in the screening based on visual inspection. If an extra examination is indicated, volumetry (Boffi Ribeiro et al., 2010) or circumferential measurements (Hüter-Becker and Dölken, 2011) is applied in the supplementary testing.

Some limitations are apparent. We may have omitted measures in the systematic literature review since we sometimes screened a $30 \%$ random sample of abstracts and we only searched for studies published in English or German. In addition, we might have missed recently developed measures as we performed the search on studies published between
2007 and 2012. We did not select a representative sample of clinical experts to attend the conference. However, all clinical experts were working at hand trauma centres certified by the Federation of the European Societies for Surgery of the Hand. We could have retrieved slightly varying measures from the expert survey if we included a representative sample of experts. The development of the ICF Hand $A$ and the decision on its content were predominantly a national effort. Involving an international perspective le.g. in the expert surveyl could have led to different results. However, the measures included in the ICF Hand are internationally established and have been frequently reported upon within the international scientific lit-

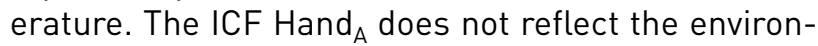
ment's positive or negative impact on a patient's situation, as originally described in the ICF. Instead, patients' future needs (e.g. for medical devices) are considered, which appeared to be more appropriate for developing an optimized and individually adapted rehabilitation strategy. For the screening of five activities and participation aspects, for example hand and arm use or interpersonal interactions and relationships, specific performance tasks and standardized questions were added to the ICF Hand A $_{\text {. These tasks }}$ and questions refer to the content and definitions in the ICF, but require explicit validation for the future use of the ICF Hand . $_{\text {. }}$

\section{Conclusion}

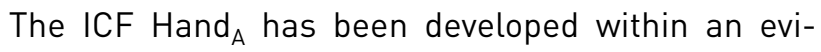
dence-based, decision-making process. It provides a consensus on which internationally established measures to use in order to systematically assess functioning in patients with hand injuries and disorders. Standardized data on patients' functioning could be generated in clinical practice and research

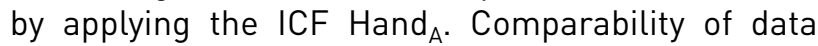
across clinical studies would be facilitated along with professional exchange among experts in different hospitals and fields of specialization.

Acknowledgements Our special thanks go to the participants of the national survey and also to Kathrin Braitmayer, Michaela Kirschneck and Heinrich Gall for their invaluable support in performing the national survey and in preparing and organizing the consensus conference.

Consortium Lighthouse Project Hand: Claudia Best, Birgit Dölz, Nicole Drummer, Andrea Franz, Kristian Weißenberg, Wolf-Lutz Müller, Frank Siemers (BG Klinikum Bergmannstrost Halle), Christian Betz, Alexander Franz, Götz Hanebuth, Michael Sauerbier (BG Unfallklinik Frankfurt am Main), Berthold Bickert, Victoria Struckmann, 
Ulrich Kneser (BG Klinik Ludwigshafen), Adrien Daigeler, Oliver Höffken, Christoph Sachs, Marcus Lehnhardt (BG Universitätsklinikum Bergmannsheil Bochum), Sonja Bonness, Caroline Dereskewitz, Katja Freund, KlausDieter Rudolf, Claudia Schröder-Kraft, Anja Thielitz, Swen Malte John (BG Klinikum Hamburg), Sabine Drisch, Christoph Heidenreich, Verena Stein, Nils Baas (BG Unfallklinik Murnau), Pattariya Jänsch, Maryam Wickert, Andreas Eisenschenk (Unfallkrankenhaus Berlin), Florian Goehtz, Natascha Weihs, Jörg van Schoonhoven (Rhön Klinikum AG Bad Neustadt a. d. Saale), Burkhard Heitmann, Detlef Schreier, Heinz Herbert Homann (BG Klinikum Duisburg), Andreas Nusche, Stéphane Stahl, Hans-Eberhard Schaller (BG Klinik Tübingen).

Supplementary material Supplementary material is available at: http://journals.sagepub.com/doi/suppl/10.1177/ 1753193417706248.

Declaration of Conflicting Interests The authors declared no potential conflicts of interest with respect to the research, authorship, and/or publication of this article.

Funding The author(s) disclosed receipt of the following financial support for the research, authorship, and/or publication of this article: This work was supported by the German Statutory Accident Insurance Igrant number FR189). The responsibility for the content of the article lies with the authors.

Ethical standard The study was performed according to the principles of the Declaration of Helsinki 1996. All relevant study materials were approved by the ethics committee of the Ludwig-Maximilians-Universität (LMU) München, Germany.

\section{References}

Basler HD. Akutschmerztherapie in Pädiatrie und Geriatrie - Schmerzmessung: Welche Schmerzskala bei welchem Patienten? Anästh Int Notfall Schmerzther. 2011, 46: 334-42.

Biernacki P, Waldorf D. Snowball sampling: problems and techniques of chain referral sampling. Sociological Meth Res. 1981, 10: 141-63.

Birch A, Nuttall D, Stanley JK, Trail IA. The outcome of wrist surgery: what factors are important and how should they be reported? J Hand Surg Eur. 2011, 36: 308-14.

Boffi Ribeiro RC, Fonseca Lima SM, Gomes Carreira AC, Masiero $D$, Chamlian TR. Inter-tester reliability assessment of the volumetric measurement of the hand in subjects without any changes in their upper extremities. Acta Fisiatr. 2010, 17: 3-7.

Bryant D, Fernandes N. Measuring patient outcomes: a primer. Injury. 2011, 42: 232-5.

Changulani M, Okonkwo U, Keswani T, Kalairajah Y. Outcome evaluation measures for wrist and hand: which one to choose? Int Orthop. 2008, 32: 1-6.

Chung KC, Pillsbury MS, Walters MR, Hayward RA. Reliability and validity testing of the michigan hand outcomes questionnaire. J Hand Surg Am. 1998, 23: 575-87

Dubert T. Outcome measurements in hand and upper limb surgery. Chirurgie de la Main. 2014, 33: 235-46.

Goldhahn J, Angst F, Simmen BR. What counts: outcome assessment after distal radius fractures in aged patients. J Orthop Trauma. 2011, 16: 58-66.
Grüne S (Ed.) Anamnese - Untersuchung - Diagnostik. Heidelberg, Springer, 2007.

Hudak PL, Amadio PC, Bombardier C. Development of an upper extremity outcome measure: the DASH (disabilities of the arm, shoulder and hand). Am J Ind Med. 1996, 29: 602-8.

Hüter-Becker A, Dölken M (Eds.) Untersuchen in der physiotherapie. Stuttgart, Thieme, 2011.

Isernhagen S. Functional capacity evaluation: rationale, procedure, utility of the kinesiophysical approach. J Occupat Rehabil. 1992, 2: 157-68.

Kroenke K, Spitzer RL, Williams JB. The Patient Health Questionnaire-2: validity of a two-item depression screener. Med Care. 2003, 41: 1284-92.

Kroenke K, Spitzer RL. The PHQ-9: a new depression diagnostic and severity measure. Psychiatric Annals. 2002, 32: 509-21.

Kroenke K, Spitzer RL, Williams JB, Monahan PO, Lowe B. Anxiety disorders in primary care: prevalence, impairment, comorbidity, and detection. Ann Internal Med. 2007, 146: 317-25.

Kroenke K, Spitzer RL, Williams JB. The PHQ-9: validity of a brief depression severity measure. J Gen Intern Med. 2001, 16: 606-13.

Kus S, Dereskewitz C, Wickert M et al. Validation of the comprehensive international classification of functioning, disability and health (ICF) core set for hand conditions. Hand Therapy. 2011, 16: 58-66.

Kus S, Oberhauser C, Cieza A. Validation of the brief international classification of functioning, disability, and health (ICF) core set for hand conditions. J Hand Ther. 2012, 25: 274-86; quiz 87.

Kus S, van de Ven-Stevens LA, Coenen M, Berno S, Kollerits B, Cieza A. What is our knowledge of functioning and disability in hand conditions based on? Arch Phys Med Rehabil. 2011a, 92: 1326-32.

Lundborg G, Rosen B. The two-point discrimination test - time for a re-appraisal? J Hand Surg Br. 2004, 29: 418-22.

MacDermid JC. Patient-reported outcomes: state-of-the-art hand surgery and future applications. Hand Clin. 2014, 30 : 293-304.

Maercker A, Schützwohl M. Erfassung von psychischen Belastungsfolgen: Die Impact of Event Skala-revidierte Version (IES-R). Diagnostica. 1998, 44: 130-41.

Marks M, Schoones JW, Kolling C, Herren DB, Goldhahn J, Vliet Vlieland TP. Outcome measures and their measurement properties for trapeziometacarpal osteoarthritis: a systematic literature review. J Hand Surg Eur. 2013, 38: 822-38.

Mathiowetz V, Weber K, Volland G, Kashman N. Reliability and validity of grip and pinch strength evaluations. J Hand Surg Am. 1984, 9: 222-6.

Metcalf C, Adams J, Burridge J, Yule V, Chappell P. A review of clinical upper limb assessments within the framework of the WHO ICF. Musculoskeletal Care. 2007, 5: 160-73.

Moher D, Liberati A, Tetzlaff J, Altman DG. Preferred reporting items for systematic reviews and meta-analyses: the PRISMA statement. J Clin Epidemiol. 2009, 62: 1006-12.

Moriya T, Aoki M, Iba K, Ozasa Y, Wada T, Yamashita T. Effect of triangular ligament tears on distal radioulnar joint instability and evaluation of three clinical tests: a biomechanical study. J Hand Surg Eur. 2009, 34: 219-23.

$\mathrm{Ng} \mathrm{CL}, \mathrm{Ho}$ DD, Chow SP. The Moberg pickup test: results of testing with a standard protocol. J Hand Ther. 1999, 12: 309-12.

Rosales RS. Clinical research in hand surgery. J Hand Surg Eur. 2015, 40: 546-8.

Rudolf K-D, Kus S, Chung KC, Johnston M, LeBlanc M, Cieza A. Development of the international classification of functioning, disability and health core sets for hand conditions-results of the World Health Organization international consensus process. Disabil Rehabil. 2012, 34: 681-93. 
Spitzer RL, Kroenke K, Williams JW, Löwe B. A brief measure for assessing generalized anxiety disorder: the GAD-7. Arch Internal Med. 2006, 166: 1092-7.

Strauch B, Lang A, Ferder M, Keyes-Ford M, Freeman K, Newstein D. The ten test. Plast Reconstr Surg. 1997, 99: 1074-8.

Tiffin J, Asher EJ. The Purdue pegboard; norms and studies of reliability and validity. J Appl Psychol. 1948, 32: 234-47.

van de Ven-Stevens LA, Kus S, Graff M, Geurts AC. Which assessment tools address the categories of the brief ICF core set for hand conditions? Hand Therapy. 2015, 20: 75-87.

van de Ven-Stevens LA, Munneke M, Terwee CB, Spauwen PH, van der Linde $\mathrm{H}$. Clinimetric properties of instruments to assess activities in patients with hand injury: a systematic review of the literature. Arch Phys Med Rehabil. 2009, 90 : 151-69.
Velstra I-M, Ballert CS, Cieza A. A systematic literature review of outcome measures for upper extremity function using the international classification of functioning, disability, and health as reference. PM\&R. 2011, 3: 846-60.

Waldner-Nilsson B (ed.) Handrehabilitation. Für Ergo- und Physiotherapeuten; Band 1: Grundlagen, Erkrankungen. Heidelberg, Springer, 2009, No. 2.

Weinstein S. Fifty years of somatosensory research: from the Semmes-Weinstein monofilaments to the Weinstein Enhanced Sensory Test. J Hand Ther. 1993, 6: 11-22; discussion 50.

Weiss DS, Marmar CR. The impact of event scale-revised. Assessing Psychological Trauma and PTSD. 1997, 2: 168-89.

World Health Organization. International classification of functioning, disability and health (ICF). Geneva, World Health Organization, 2001. 